\title{
Microstructural Observations on the Pore Structure Development In Carbon-Carbon Composites During Processing
}

James H. Steele Jr.,

Consultant, 2019 Nova Road, Pine CO 80470

Carbon-carbon $(\boldsymbol{C}-\boldsymbol{C})$ composites consist of highly ordered graphite fibers embedded in a carbon matrix with an open pore structure that is developed during processing. They are produced by building up a carbon matrix on a fiber preform through a series of impregnation and pyrolysis steps. The pore structure, which is inherent in $\boldsymbol{C}-\boldsymbol{C}$ composites, changes during each processing step. Microstructural observations will include: 1) $\boldsymbol{S E M}$ backscatter $(\boldsymbol{B S E})$ images of the microstructures. 2) $\boldsymbol{S E M}$ line scan analysis of the porosity. 3) Mercury intrusion results on the pore structure.

The porous $\boldsymbol{C}-\boldsymbol{C}$ samples were mounted in a liquid Bi-Sn alloy at $145^{\circ} \mathrm{C}$ and $8 \mathrm{ksi}$, and cooling the mount while maintaining the pressure[1]. This mounting technique, which fills open porosity larger than 20nm, allows $\boldsymbol{C}$ - $\boldsymbol{C}$ samples to be ground and polished by standard methods where diamond paste is used for final polishing. Argon ion etching may be used, if needed, to remove flowed layers from the polished sections. $\boldsymbol{B S E}$ imaging can then be applied to examine the microstructures, as illustrated in Figures 1 and 2, which show both the macro and micro-scale porosity that is observed.

Processing steps for the $\boldsymbol{C}$ - $\boldsymbol{C}$ composites examined are listed in Table 1, along with stereological estimates of the volume fraction $(\boldsymbol{V} \boldsymbol{v})$ of open porosity, and mean chord length within the pores. These estimates were obtained via line scan analysis using a computer controlled $\boldsymbol{S E M}$. The two different values for mean chord length were obtained from line scans perpendicular and parallel to the direction of the fiber weave. These two values indicate the extent of anisotropy that was observed in the pore size measurements. Estimated values for average density of the solid carbon phases, which were obtained from apparent density and the estimated solid $\boldsymbol{V} \boldsymbol{v}$ (1- $\boldsymbol{V} \boldsymbol{v}$ (pores)), are also listed in Table 1.

Chord length distributions were also obtained from line scan data on the open porosity. An example of a measured chord length distribution is provided in Figure 3 for the pyrolized sample. Chord length distribution functions have recently been discussed in terms of their basic importance in transport problems[2], and in 3-d reconstruction of porous media[3]. Pore size distributions were also obtained from mercury intrusion results on the $\boldsymbol{C}$ - $\boldsymbol{C}$ composites[4]. These results, which are presented in Figure 4, illustrate how the fine porosity that was created during carbonization (shown in Figure 1) evolves through the remaining processing steps.

\section{References}

[1] E.T. Rose and L.F. DeRoos, Microstructural Science, Vol. 1 (1972) 275.

[2] S. Torquato and B. Lu, Phys. Rev. E, 47 (1993) 2950.

[3] A.P. Roberts and M. Tuebner, Phys. Rev. E, 51 (1995) 4141.

[4] J. Van Brakel et al., Powder Technology, 29 (1981) 1.

[5] The Support of Armco Inc (1982-1984), and Hitco Inc for $\boldsymbol{C}$ - $\boldsymbol{C}$ Composite Material, and R.J. Lee Associates for use of the Computer Controlled SEM, are hereby acknowledged. 


\begin{tabular}{|c|c|c|c|c|c|}
\hline$\underline{\text { Sample }}$ & $\underline{\text { Table } I}=$ Processing Treatment & $\frac{V v(\text { pores })}{\%}$ & \multicolumn{2}{|c|}{$\underline{M F P} \mu m$} & $\frac{\text { Solid Density }}{\mathrm{gm} / \mathrm{cc}}$ \\
\hline GAC38 & Phenolic resin in Carbon Fiber Preform & $27 \pm 5$ & $53 \pm 5$ & $30 \pm 2$ & $1.80 \pm 0.1$ \\
\hline GAC39 & Carbonize @ $540^{\circ} \mathrm{C}$ in Controlled atm. & $40 \pm 4$ & $39 \pm 2$ & $16 \pm 1$ & $1.85 \pm 0.1$ \\
\hline GAC40 & Pyrolysis@2200 ${ }^{\circ} \mathrm{C}$ in Controlled atm. & $54 \pm 4$ & $51 \pm 3$ & $37 \pm 2$ & $2.1 \pm 0.1$ \\
\hline GAC41 & $1^{\text {st }}$ CVD from hydro-carbon gas. & $44 \pm 3$ & $48 \pm 2$ & $37 \pm 2$ & $2.1 \pm 0.1$ \\
\hline GAC42 & $2^{\text {nd }}$ CVD from hydro-carbon gas & $33 \pm 3$ & $32 \pm 2$ & $21 \pm 1$ & $2.0 \pm 0.1$ \\
\hline GAC43 & Final Pyrolysis@2100-2200 C & $41 \pm 3$ & $38 \pm 2$ & $25 \pm 1$ & $2.1 \pm 0.1$ \\
\hline
\end{tabular}

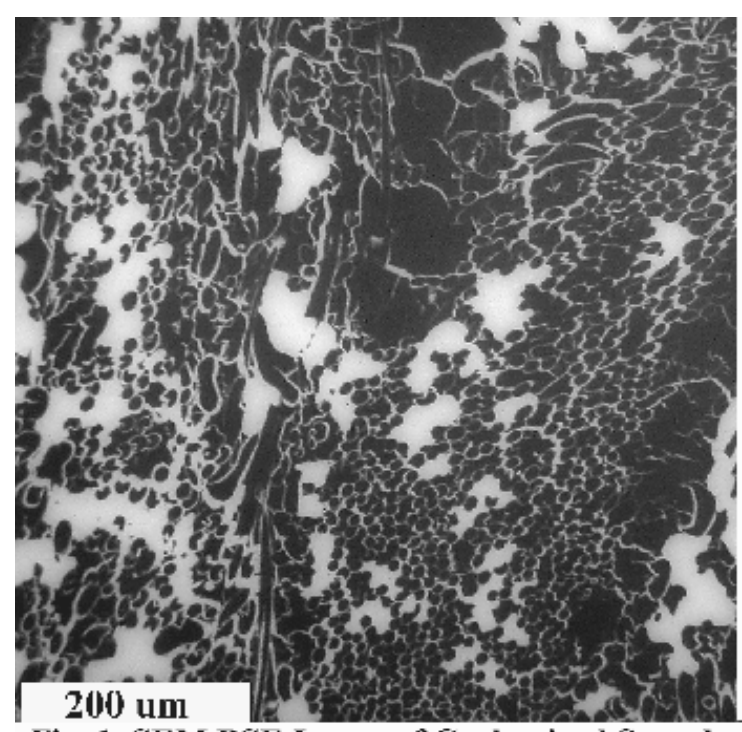

Fig. 1. SEM BSE Image of Carbonized Sample.

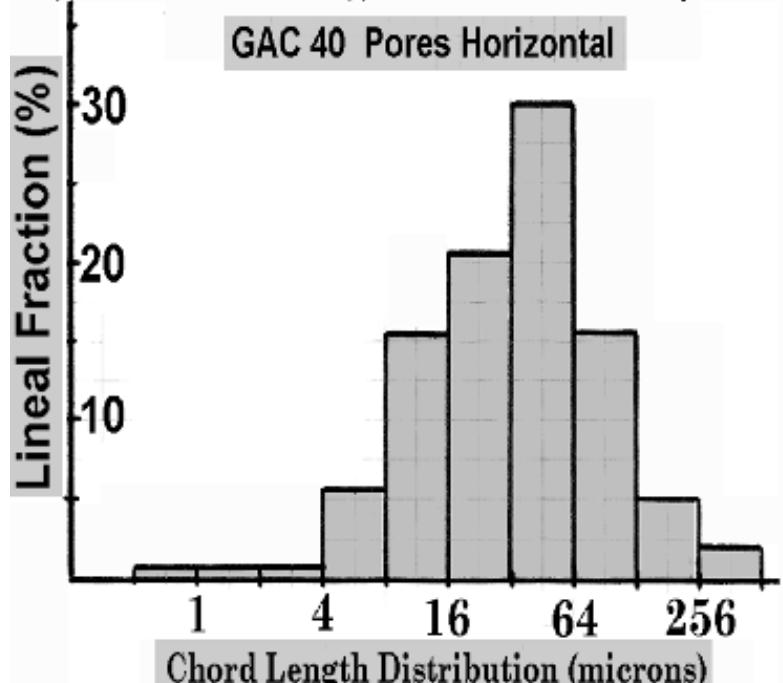

Fig. 3. Measured Chord Length Distribution.

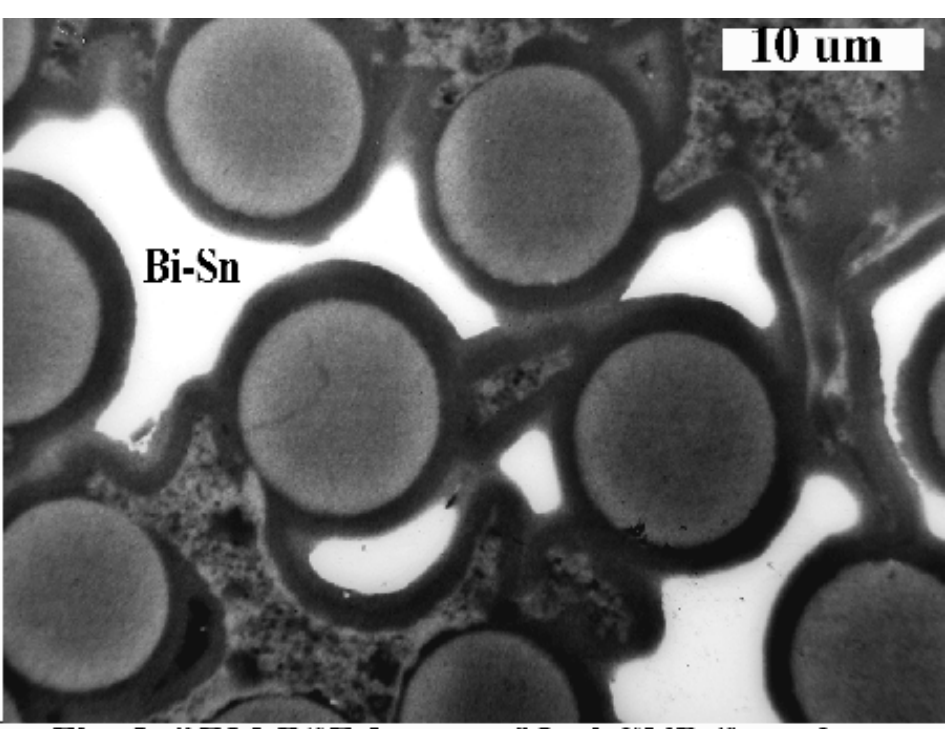

Fig. 2. SEV BSE Image of 2nd CVD Sample.

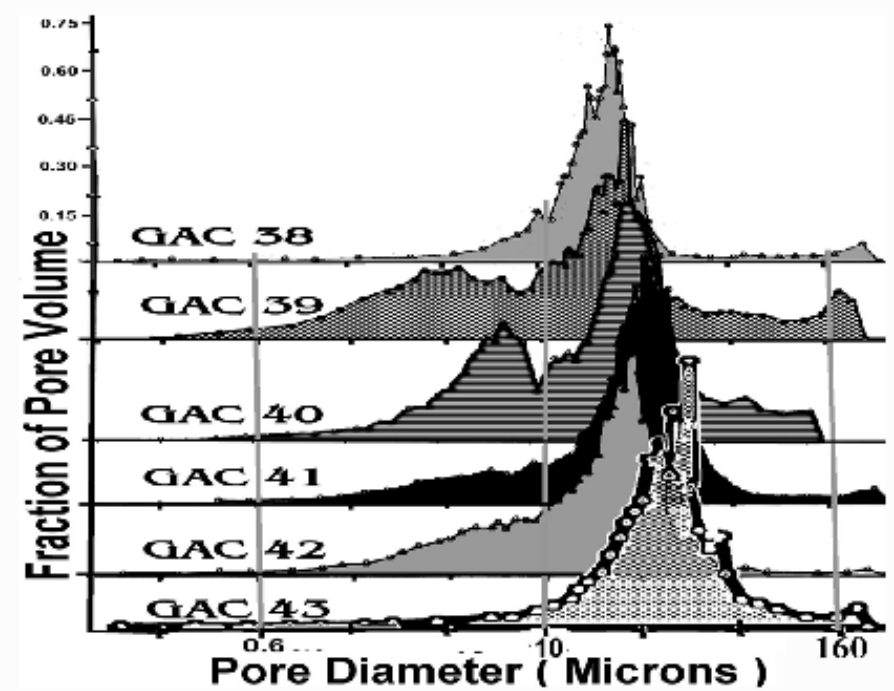

Fig. 4. Mercury Intrusion Pore Size Distributions. 